\title{
The impact of solution representations on heuristic net present value optimization in discrete time/cost trade-off project scheduling with multiple cash flow and payment models
}

\author{
Pieter Leyman ${ }^{1,2}$, Niels Van Driessche ${ }^{2}$, Mario Vanhoucke ${ }^{2,3,4}$, and Patrick De Causmaecker ${ }^{1}$ \\ ${ }^{1}$ KU Leuven KULAK, Department of Computer Science, CODeS, Etienne Sabbelaan 53, 8500 Kortrijk \\ (Belgium),pieter.leyman@kuleuven.be, patrick.decausmaecker@kuleuven.be \\ ${ }^{2}$ Faculty of Economics and Business Administration, Ghent University, Tweekerkenstraat 2, 9000 Ghent (Belgium), \\ mario.vanhoucke@ugent.be \\ ${ }^{3}$ Technology and Operations Management Area, Vlerick Business School, Reep 1, 9000 Ghent (Belgium) \\ ${ }^{4}$ UCL School of Management, University College London, 1 Canada Square, London, E14 5AA (United Kingdom)
}

\begin{abstract}
The goal of this paper is to investigate the impact of different solution representations, as part of a metaheuristic approach, on net present value optimization in project scheduling. We specifically consider the discrete time/cost trade-off problem with net present value optimization and apply three payment models from literature. Each of these models determines the timing and size of cash flows from the contractor's viewpoint. The contribution of this paper to literature is twofold.

First, we include cash flow distribution variants in the payment models, to also distinguish between different manners in which value is created and costs are incurred, as part of a general model for the contractor's cash flow management. This general model is developed in order to explicitly include the progress of activities in the determination of the timing and size of payments to the contractor, which is currently lacking in literature.

Second, we employ an iterated local search framework to compare different solution representations and their corresponding local search and repair heuristics. The goal is to unambiguously show that the choice of a solution representation deserves a fair amount of attention, alongside the selection of appropriate diversification and intensification operators, even though this is not always the case in literature. Each part of the proposed algorithm is validated on a large dataset of test instances, generated to allow for a broad comparison of the solution representations. Our results clearly quantify the statistically significant differences between three types of representations for the project scheduling problem under study.

Keywords: Project scheduling, Net present value, Discrete time/cost trade-off, Heuristics, Solution representations
\end{abstract}




\section{Introduction}

Metaheuristic algorithms focus on finding good solutions within reasonable computation time, but offer no guarantee of obtaining the optimal solution. These techniques are hence better suited to real-life problems than exact methods, because they allow for quick decision making. Metaheuristics furthermore compose frameworks that provide guidelines to design heuristic solution techniques (Sörensen and Glover, 2013). A multitude of metaheuristic frameworks exist, each of which are based on a wide variety of principles or analogies (see e.g. Gendreau and Potvin, 2010 and Burke and Kendall, 2014 for recent overviews). An important part of each metaheuristic is the solution representation or encoding, since a metaheuristic does not operate on a solution itself, but rather on the representation of that solution (Sörensen and Glover, 2013). We contribute to metaheuristic research by analyzing and comparing different solution representations and their corresponding local searches and repair methods, as part of a component-based view on metaheuristics (Sörensen, 2015). Furthermore, we extend existing work in project scheduling literature by introducing a general cash flow management model. In the remainder of this section, we first discuss different solution representations used in a project scheduling context, after which an overview of existing work on the specific project scheduling problem discussed is provided.

\subsection{Solution representations in project scheduling}

In project scheduling literature, a commonly used representation for (meta)heuristic optimization is the activity list (AL), which contains all activity numbers in a precedence feasible order, i.e. each activity occurs in the list after all of its predecessors. The AL is subsequently translated into a project schedule by making use of a schedule construction algorithm, of which the serial schedule generation scheme (Kelley, 1963) is the most commonly used. Depending on the objective, a local search can subsequently be applied to improve the objective function value of the schedule. Whereas intensification and diversification techniques have received considerable attention, little focus has been on the use of an appropriate solution representation, as part of a metaheuristic solution approach.

Kolisch and Hartmann (1999) compare several representations for the resource-constrained project scheduling problem (RCPSP), of which the goal is to minimize the project duration while satisfying precedence and renewable resource restrictions. The authors conclude that the AL and random key (RK) are most commonly used. Valls et al. (2003) propose to use a topological ordering (TO) representation to mediate shortcomings of the RK and AL representations, and conclude that existing results for the RCPSP can be improved. Leyman (2016) is the first to consider the slack list (SL) representation. The author shows that the SL significantly outperforms the commonly used finish time list (FTL) representation on the discrete time/cost trade-off problem with net

present value optimization. Li et al. (2018) combine the SL with a TO to represent solutions for 
the resource leveling problem with generalized precedence relations, and show that the combination leads to high quality schedules.

Aside from the aforementioned works, to the best of our knowledge, no research has been done on the impact of solution representations in a project scheduling context. In particular, in case the initial schedule can be improved by delaying activities, such as in case of a net present value (NPV) objective (Leyman and Vanhoucke, 2015), the impact of solution representations has not been studied. To mediate this gap in literature, we propose to evaluate the impact of several solutions representations as part of an iterated local search algorithm. We apply the algorithm on the discrete time/cost trade-off problem with NPV optimization and three payment models.

\subsection{Discrete time/cost trade-off in project scheduling}

An extensively examined problem in project scheduling literature revolves around the trade-off between different activity modes. These modes correspond with different values for each activity's duration and cost, with lower (higher) durations typically incurring a higher (lower) cost. If this trade-off furthermore corresponds with a discrete non-increasing function, we talk about the discrete time/cost trade-off problem (DTCTP). Three different problem variations of the DTCTP exist, depending on the objective. The deadline problem minimizes the total project cost subject to a fixed deadline, whereas the budget problem minimizes project duration given a total cost restriction. The final objective is to construct the efficient frontier of all time/cost combinations with feasible project durations.

An important distinction has to be made between the DTCTP on the one hand, and multi-mode scheduling as an extension to the RCPSP on the other hand. In both problems, a single mode has to be selected for each activity, in which the activity is scheduled without preemption and any mode changes. Different resource restrictions, however, apply for both problems. The multimode RCPSP includes multiple renewable and non-renewable resources, whereas the DTCTP only employs a single non-renewable resource, which constitutes the available budget (Hartmann and Briskorn, 2010). For overviews of the DTCTP, we refer to Vanhoucke and Debels (2007) and Hazir et al. (2010), whereas a recent overview on the multi-mode RCPSP can be found in Van Peteghem and Vanhoucke (2011).

In this paper, we go into detail about an extension of the deadline variant of the DTCTP, namely the DTCTP with NPV optimization (DTCTP-NPV). Erengüç et al. (1993) were the first to discuss the DTCTP-NPV, as a combination of the DTCTP and the payment scheduling problem. Both cash in- and outflows are associated with events in the research. The model of Erengüç et al. (1993) is extended by Vanhoucke and Debels (2007), by assigning cash outflows with arcs and cash inflows with events in an activity-on-the-arc (AoA) network representation. The authors obtain near-optimal solutions for the problem by making use of dynamic programming and a metaheuristic algorithm. A bonus-penalty structure is included in the DTCTP-NPV by He and Xu 
(2008) with both cash in- and outflows again at event completion. The goal is to balance the client and contractor NPV by including a trade-off mechanism. Their model is tested with a simulated annealing algorithm on an example from literature. He et al. (2009a) use a similar simulated annealing approach to discuss the client's perspective in detail, whereas He et al. (2009b) propose three different payment models for the cash flows from the client to the contractor. These payment models determine the timing and size of payments to the contractor, based on the schedule used. As a result, links are imposed between the activity finish times on the one hand and the payment times and sizes on the other hand. The authors test several algorithms and conclude that the nested loop simulated annealing metaheuristic outperforms the others for all payment models. He et al. (2012) build upon their earlier work by additionally imposing capital restrictions, which state that the cash position of the contractor cannot become negative during the project. Financing costs, which are to be distributed evenly over the client and contractor, are included by He et al. (2014). The goal is to find a schedule agreeable for both parties, given the payment models. Leyman (2016) compares different solution representations and uses an activity-on-the-node (AoN) network representation. Cash outflows are discounted to the activity finish times, but cash inflows are accrued during the runtime of each activity instead of at the finish time. He et al. (2017) minimize the contractor's cash flow gap between cash in- and outflows in an AoA network, instead of maximizing the project NPV. Several conclusions are drawn with respect to the impact of data parameters on the cash flow gap and allow for decision making support. Finally, Ning et al. (2017) include uncertainty in the activity durations and aim to generate robust baseline schedules for the DTCTP-NPV.

We chose the DTCTP-NPV to study the impact of different solution representations for the following reasons:

- Based on the overview above, we can conclude that the DTCTP-NPV is a relatively new problem in literature, in particular with the inclusion of different payment models. Especially the limited treatment of cash flow models (Section 2.1) and the usage of mostly the AoA network representation merit further research.

- The FTL is commonly used for DTCTP-NPV, but as shown by Leyman (2016) it does not necessarily lead to the best performance, which justifies further investigation.

- Algorithms for the DTCTP-NPV generally do not use any or few local search heuristics. If, on the contrary, renewable resources are included, as is the case for the resource-constrained project scheduling problem with net present value optimization (RCPSPDC), local search techniques contribute greatly to algorithm performance (Leyman and Vanhoucke, 2015). Based on the solution representation used, we wish to examine whether local searches can have an added value for the DTCTP-NPV as well, and quantify these differences in performance.

In light of this, we believe it would be interesting to evaluate the effect of different solution representations on the DTCTP-NPV with several cash flow and payment models. Our contribution 
to literature can be summarized as follows:

- We propose a general model for a contractor's cash flow management, which includes activity progress to decide upon timing and size of received payments (Section 2.1). The model furthermore allows for different ways of value creation for the client and of cost incurrence by the contractor.

- We demonstrate the importance of solution representations as part of an iterated local search metaheuristic and their impact on algorithm performance (Sections $3.1 \& 3.2$ ). Our results recognize the relevance of including both schedule and priority information into the solution representation (Section 4.3).

\subsection{Paper overview}

The remainder of this paper is organized as follows. In Section 2, the problem definition of the DTCTP-NPV is discussed, along with the three payment models used. We analyze the NPV optimization problem from the contractor viewpoint, and assume that any negotiations between the client and contractor have been completed. We additionally present different cash flow models, which determine the progress of an activity in terms of cash flows. The distinction is made between cash in- and outflows to study the impact of both on the project NPV. In Section 3, the iterated local search algorithm is explained along with the application of different solution representations for the DTCTP-NPV. We include local search and repair methods to both ensure feasibility and to improve the project NPV. In Section 4, we discuss the results of the algorithm and compare the different representations with each other. We finish with conclusions and recommendations for future research in Section 5 .

\section{The discrete time/cost trade-off problem with net present value optimization and different payment models}

A project can be represented by a directed graph or network $G(N, A)$ with $N$ the nodes or project activities and $A$ the arcs or precedence relations between individual activities. We use an AoN representation and employ a time lag of zero for the precedence relations. As a result, an activity can start as soon as all of its predecessors have finished. All of the activities $i(i \in N=\{1, \ldots,|N|\})$ have a mode $m\left(m \in M_{i}=\left\{1, \ldots,\left|M_{i}\right|\right\}\right)$, with which corresponds a duration $d_{i m}$ and a cost $c_{i m \text {,out }}$. Furthermore, the activity durations are modeled as discrete non-increasing functions of the costs associated with them, i.e. $d_{i 1}>d_{i 2}>\ldots>d_{i\left|M_{i}\right|}$ and $c_{i 1 \text {,out }}<c_{i 2 \text {,out }}<\ldots<c_{i\left|M_{i}\right| \text {,out }}$. Start and end dummy nodes, 0 and $n+1$ respectively, are used to determine the start and end of the project. Both nodes have only one mode, with a duration and cost equal to zero. A project deadline $\delta_{|N|+1}$ is also included. The decisions to be made concern the activity finish times $f_{i}\left(f_{i} \geq 0\right)$ and the 
selected modes for each activity, represented by $x_{i m}\left(x_{i m} \in\{0 ; 1\}, \sum_{m=1}^{\left|M_{i}\right|} x_{i m}=1\right) . x_{i m}$ equals 1 if activity $i$ is executed in mode $m$, and 0 otherwise.

The deadline variant of the discrete time/cost trade-off problem with NPV maximization optimizes project NPV with precedence and deadline restrictions. The cash outflows or costs $c_{i m \text {,out }}$ of an activity $i$ executed in mode $m$, along with the cash inflows $c_{i \text {,in }}$ for each activity $i$, constitute the necessary cash flows to model the problem. Important to note is that whereas the cash outflows depend on the selected activity modes, the cash inflows are independent of the employed modes. In literature, both cash flows are typically assumed to occur either at the start or at the end of an activity. We, however, introduce a general model for both cash in- and outflows, based on the capital management model of Leyman and Vanhoucke (2017).

In the remainder of this section, we first discuss the cash flow models used, before going into detail about payment models. The crucial difference between both types of models can be summarized as follows. Cash flow models determine the costs accrued by the contractor and the value created for the client per activity, but do not necessarily correspond with actual payments to the contractor. Payment models on the contrary set the occurrence time and size of payments to the contractor (cash inflows), depending on the progress of the project with respect to payment thresholds. Below, we provide a summary of these payment models, clarifying how these fit with the proposed cash flow models, and supporting a proper understanding of the problem as a whole. An overview of the notations used as part of the models in this section, is shown in Table 1.

\subsection{Cash flow models}

A general model for cash outflows, which influence the capital availability throughout the project, was proposed by Leyman and Vanhoucke (2017). The cash flow models discussed here extend upon this work, by including cash inflows as well, in order to obtain an overall model for the cash flows in a project. No capital restrictions are, however, employed in this work, to both allow for a clearer focus on the interaction between cash in- and outflows, and with the payment models of Section 2.2 .

The progress of an activity in terms of costs (cash outflows) or value for the client (cash inflows) can be modeled based on parameters $w_{i m t}\left(w_{i m t} \in[0 ; 1]\right)$ and $v_{\text {imt }}\left(v_{\text {imt }} \in[0 ; 1]\right)$ respectively. These parameters hold the fraction of the cost incurred or value created at time $f_{i}-d_{i m}+t$ for each activity $i$ in mode $m$, and are set in advance. $\sum_{t=0}^{d_{i m}} w_{i m t}=1$ and $\sum_{t=0}^{d_{i m}} v_{i m t}=1$ for all activities and for all modes, to ensure that the entire cash flows are assigned. In doing so, we distribute the costs and value of each activity over its duration, which depends on the selected activity mode, in a predefined manner. If we assume payments can occur at every time instance, then the NPV of the project can be calculated as follows: 


\begin{tabular}{ll}
\hline Notation & Definition \\
\hline$A$ & Set of arcs \\
$N$ & Set of activities \\
$i$ & Activity index \\
$M_{i}$ & Set of modes of activity $i$ \\
$m$ & Mode index \\
$d_{i m}$ & Duration of activity $i$ with mode $m$ \\
$c_{i m, \text { out }}$ & Cost/cash outflow of activity $i$ with mode $m$ \\
$c_{i, \text { in }}$ & Cash inflow of activity $i$ \\
$f_{i}$ & Finish time of activity $i$ \\
$x_{i m}$ & 1 if activity $i$ is executed in mode $m, 0$ otherwise \\
$t$ & Time index \\
$w_{i m t}$ & Fraction of cost incurred at time $f_{i}-d_{i m}+t$ for activity $i$ in mode $m$ \\
$v_{i m t}$ & Fraction of value created at time $f_{i}-d_{i m}+t$ for activity $i$ in mode $m$ \\
$\mathrm{TC}_{t}$ & Total cost incurred at time $t$ \\
$\mathrm{CF}_{\text {out }, t}$ & Total cash outflow at time $t$ \\
$\mathrm{NPV}_{\text {out }, t}$ & NPV of total cash outflow at time $t$ \\
$\mathrm{TV}_{t}$ & Total value created at time $t$ \\
$\mathrm{CF}_{\text {in }, t, \mathrm{X}}$ & Total cash inflow at time $t$ for payment model X \\
$\mathrm{NPV}_{\text {in }, t, \mathrm{X}}$ & NPV of total cash inflow at time $t$ for payment model X \\
$\delta_{|N|+1}$ & Project deadline \\
$\alpha$ & Discount rate \\
$K$ & Number of payments \\
$\theta$ & Compensation proportion \\
\hline
\end{tabular}

Table 1: Overview of notations.

$$
\mathrm{NPV}=\sum_{i=1}^{|N|} \sum_{m=1}^{\left|M_{i}\right|} \sum_{t=0}^{d_{i m}}\left(c_{i, \mathrm{in}} \cdot v_{i m t}-c_{i m, \mathrm{out}} \cdot w_{i m t}\right) \cdot e^{-\alpha \cdot\left(f_{i}-d_{i m}+t\right)} \cdot x_{i m}
$$

with the exponential function used to discount cash flows to their time of receipt (cash in) or payment (cash out).

To illustrate the use of the $w_{i m t}$ and $v_{i m t}$ parameters, we employ three models, which are shown in Figure 1. The vertical axes show the total cost incurred at time $t\left(\mathrm{TC}_{t}\right)$ and the total value created at time $t\left(\mathrm{TV}_{t}\right)$ respectively, whereas the horizontal axis displays the time $t$. Model 1 and 3 can be seen as extreme cases for both the cost and value of an activity, whereas model 2 serves as a middle ground and a representation of all model variations between both extremes. The three models can be summarized along the following lines:

- Model 1: $w_{i m 0}=1\left(v_{i m 0}=1\right)$ : the entire cost (value) is incurred (created) at the start of the activity. As a result, increases in $\mathrm{TC}_{t}\left(\mathrm{TV}_{t}\right)$ in the left graphs of Figure 1 occur at the start of the activity $i$.

- Model 2: $w_{i m 0 \ldots d_{i m}-1}=1 / d_{i m}\left(v_{i m 1 \ldots d_{i m}}=1 / d_{i m}\right)$ : the cost (value) is incurred (created) in a stepwise manner during the activity duration. Consider that in terms of costs, we assume the 
first part is incurred at the start of the activity $\left(w_{i m 0}=1 / d_{i m}\right)$, whereas in terms of value the first part is created after the first $1 / d_{i m}$ part has been completed $\left(v_{i m 0}=0\right)$. This is done to clearly differentiate the cash in- and outflows in this middle ground model. The resulting cash profiles are shown in the middle graphs of Figure 1.

- Model 3: $w_{i m d_{i m}}=1\left(v_{i m d_{i m}}=1\right)$ : the entire cost (value) is incurred (created) at the end of the activity. As a result, increases in $\mathrm{TC}_{t}\left(\mathrm{TV}_{t}\right)$ in the right graphs of Figure 1 occur at the end of the activity $i$.

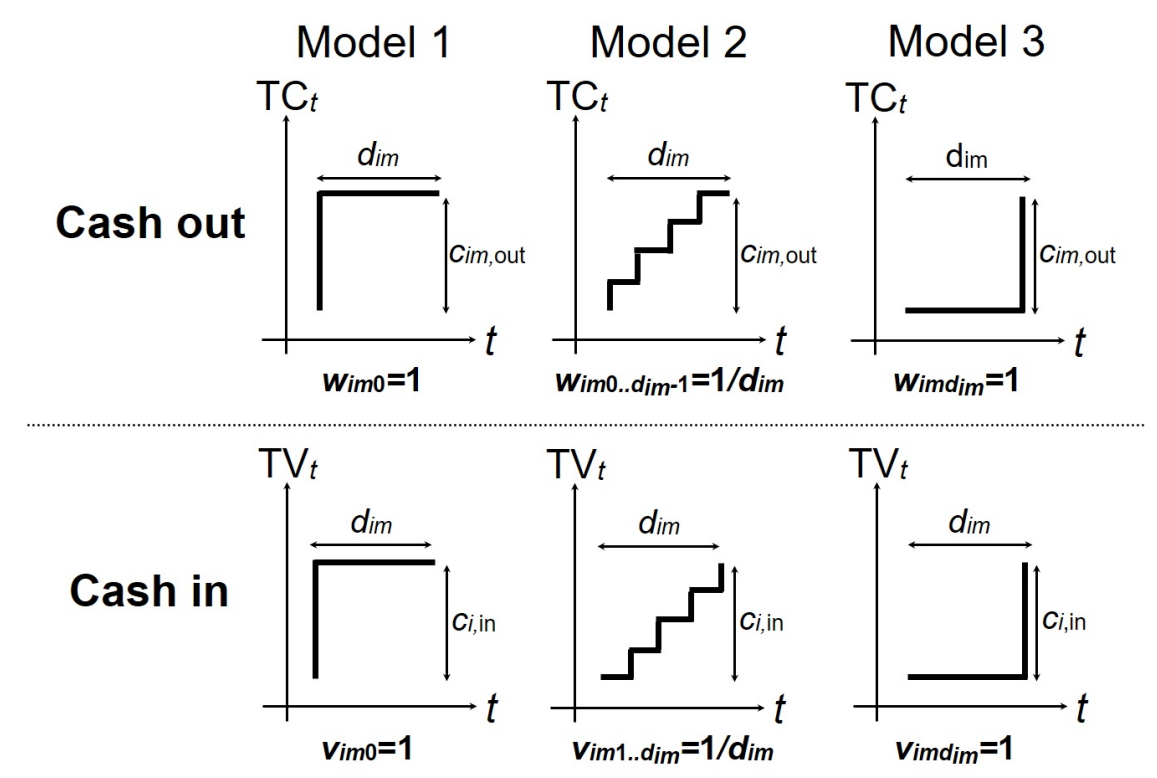

Figure 1: Applications of cash flow models.

These three models for both cash in- and outflows can furthermore be combined into nine different cases, as shown in Table 2. These cases allow for the analysis of the impact due to the progress of both cost incurred and value created together, and can be used as part of the three payment models discussed next. Earlier work in literature on the DTCTP-NPV only corresponds with cash flow cases VIII (Leyman, 2016) and IX (other work discussed in Section 1). Both are marked with * in the table. Finally, Leyman and Vanhoucke (2017) employ models III, VI and IX as part of their capital management model (marked with ${ }^{+}$in the table), but also include renewable resources. None of the other cases have been discussed to date, let alone as part of a general model.

Regarding created value, it is worth mentioning that we explicitly assume that this value is a percentage $\theta(0<\theta<1)$ of the actual created value for the client. This means that when we talk about created values, we consider the values received by the contractor, which themselves constitute a part of the value created for the client. In literature, the parameter $\theta$ is often called the compensation proportion (He et al., 2009b). 


\begin{tabular}{cc|ccc} 
& & \multicolumn{3}{|c}{ Cash in } \\
& & Model 1 & Model 2 & Model 3 \\
\hline \multirow{3}{*}{ Cash out } & Model 1 & I & II & III $^{+}$ \\
& Model 2 & IV & V & VI $^{+}$ \\
& Model 3 & VII & VIII* $^{*}$ & IX $^{*,+}$
\end{tabular}

Table 2: Nine different cash flow cases.

\subsection{Payment models}

In Section 2.1, it has been assumed that the payments of both the cash in- and outflows occur at the time the value is created and the cost is incurred respectively. This principle is retained for the cash outflows, since we do not include subcontractors in our analysis. The occurrence of payments of cash inflows on the contrary, is determined by three payment models from literature (He et al., 2009b). Specifically, this implies that the value of activities still occurs according to the cash flow cases of Section 2.1, but that the cash inflows are only received once a threshold, based on the employed payment model, is reached. As a result, the timing and size of payments depends on the payment model and the corresponding threshold used.

As stated in Section 1, we employ the contractor's point of view and assume all negotiations between the client and contractor have been completed. As a result, some restrictions have already been imposed on the timing or size of payments to the contractor. Table 3 provides an overview of the three payment models of He et al. (2009b) and their links with timing and size. In all three models the size of cash inflows depends on the total value (TV) and equals the TV at the payment time minus any previous payments. The timing on the contrary differs between the models and may be fixed in advance, may depend on the TV or may depend on the total cost (TC).

\begin{tabular}{c|ccc} 
& TBPP & PBPP & EBPP \\
\hline Timing & Fixed & TV & TC \\
Size & TV & TV & TV
\end{tabular}

Table 3: Dependencies of timing and size of cash inflows in the three payment models.

Finally, consider that the selected activity modes determine both the duration and cost for each activity. Hence, the TV and TC progression not only depend on the activity finish times (i.e. the project schedule), but also on the modes of the activities. Based on the payment model used, the timing and/or size of payments may thus be variable, in the sense that these are dependent of the selected activity modes and finish times.

We provide a summary of the three models in the remainder of this section to clearly situate them within this research, but first discuss a possible pitfall regarding network representation. 


\subsubsection{Network representation}

An important issue to be raised here concerns our choice for the AoN representation. In the DTCTP literature, it is most often assumed that projects are given in AoA format, including most papers on the DTCTP-NPV discussed in Section 1, although some authors such as Hazir et al. (2010) prefer the AoN format. In the AoA format in which the payment models were originally developed, payments are received at event occurrences, which correspond with the finish time of one or more activities. As a result, cash inflows are always received at the finish time of at least one activity. We, however, believe it would be worthwhile to employ the AoN representation, since then payments can occur at many more time units.

Allow us to illustrate this reasoning with the example of Figure 2. The top part of the figure displays the project network in AoN format with two modes per activity. We assume a project deadline of 10, which implies that mode 1 of activity 1 is infeasible. The activity modes used in this section are marked in bold in the figure, and result in finish times of 4 for activity 1 and 10 for activity 2. The bottom part of the figure displays the same example network in AoA format, with events $\mathrm{A}, \mathrm{B}$ and $\mathrm{C}$ to connect activities 1 and 2. In the AoA format, payments could occur at events $\mathrm{B}$ and $\mathrm{C}$ (not event $\mathrm{A}$ since this marks the start of the project). If we assume two payments for the project, this would imply that these payments always occur at events B and $\mathrm{C}$, and hence at times 4 and 10, given the deadline of 10 and the selected modes. In the AoN format, however, payments can but need not correspond with activity finish times, as is illustrated in the following paragraphs. This way, many more possibilities exist for the occurrence of payment times in the AoN format. As a result, the problem studied here is more difficult to optimize than the problems in previous work, while maintaining the general logic of the models of He et al. (2009b).

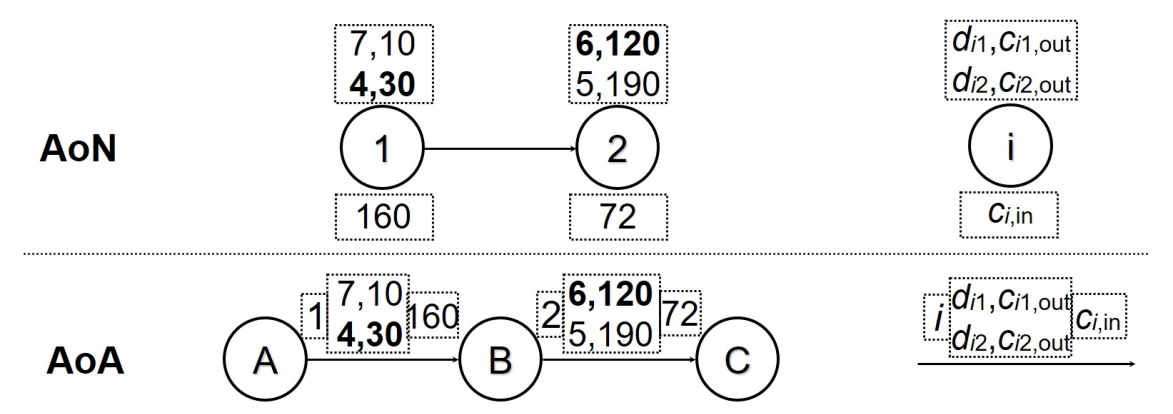

Figure 2: Example 1: Data project.

Finally, to calculate the project NPV in the example we employ cash case $\mathrm{V}$ from Table 2 for all three payment models. The total cost at each time $t$ is shown in the $\mathrm{TC}_{t}$ row in Table 4, whereas the cash outflows at each time unit are shown in the row $\mathrm{CF}_{\text {out }, t}$, which correspond with $\mathrm{TC}_{t}-\mathrm{TC}_{t-1}$. Based on a discount rate of $1 \%$, the NPV of the cash outflows is displayed in the $\mathrm{NPV}_{\text {out }, t}$ row. The occurrence of cash inflows depends on the payment model used, and is discussed 
below for each model separately, based on the total value progression displayed in the $\mathrm{TV}_{t}$ row of the table.

\begin{tabular}{r|ccccccccccccc}
$t$ & 0 & 1 & 2 & 3 & 4 & 5 & 6 & 7 & 8 & 9 & Total \\
\hline $\mathrm{TC}_{t}$ & 7.50 & 15.00 & 22.50 & 30.00 & 50.00 & 70.00 & 90.00 & 110.00 & 130.00 & 150.00 & 150.00 & 150.00 \\
$\mathrm{TV}_{t}$ & 0.00 & 40.00 & 80.00 & 120.00 & 160.00 & 172.00 & 184.00 & 196.00 & 208.00 & 220.00 & 232.00 & 232.00 \\
\hline $\mathrm{CF}_{\text {out }, t}$ & 7.50 & 7.50 & 7.50 & 7.50 & 20.00 & 20.00 & 20.00 & 20.00 & 20.00 & 20.00 & 0.00 & 150.00 \\
$\mathrm{NPV}_{\text {out }, t}$ & 7.50 & 7.43 & 7.35 & 7.28 & 19.22 & 19.02 & 18.84 & 18.65 & 18.46 & 18.28 & 0.00 & 142.02 \\
\hline $\mathrm{CF}_{\text {in }, t, \mathrm{~T}}$ & 0.00 & 0.00 & 0.00 & 0.00 & 0.00 & $\mathbf{1 7 2 . 0 0}$ & 0.00 & 0.00 & 0.00 & 0.00 & $\mathbf{6 0 . 0 0}$ & 232.00 \\
$\mathrm{NPV}_{\text {in }, t, \mathrm{~T}}$ & 0.00 & 0.00 & 0.00 & 0.00 & 0.00 & 163.61 & 0.00 & 0.00 & 0.00 & 0.00 & 54.29 & 217.90 \\
\hline $\mathrm{CF}_{\text {in }, t, \mathrm{P}}$ & 0.00 & 0.00 & 0.00 & $\mathbf{1 2 0 . 0 0}$ & 0.00 & 0.00 & 0.00 & 0.00 & 0.00 & 0.00 & $\mathbf{1 1 2 . 0 0}$ & 232.00 \\
$\mathrm{NPV}_{\text {in }, t, \mathrm{P}}$ & 0.00 & 0.00 & 0.00 & 116.45 & 0.00 & 0.00 & 0.00 & 0.00 & 0.00 & 0.00 & 101.34 & 217.80 \\
\hline $\mathrm{CF}_{\text {in }, t, \mathrm{E}}$ & 0.00 & 0.00 & 0.00 & 0.00 & 0.00 & 0.00 & $\mathbf{1 8 4 . 0 0}$ & 0.00 & 0.00 & 0.00 & $\mathbf{4 8 . 0 0}$ & 232.00 \\
$\mathrm{NPV}_{\text {in }, t, \mathrm{E}}$ & 0.00 & 0.00 & 0.00 & 0.00 & 0.00 & 0.00 & 173.28 & 0.00 & 0.00 & 0.00 & 43.43 & 216.72
\end{tabular}

Table 4: Example 1: Application of payment models.

\subsubsection{The time-based payment pattern}

The time-based payment pattern (TBPP) states that payments are due once a specified amount of time has passed since the start of the project. The threshold is set to $\left\lceil\delta_{n+1} / K\right\rceil$, with $K$ the number of payments. As a result, the payment times can be set in advance, namely before a project schedule is constructed. The payment times are furthermore set in a regular manner, namely after e.g. every 5 time units, with the final payment at the project deadline. The size of the payments equals the difference between the TV at the payment time under consideration minus all previous payments. It is worth noting that this model is essentially the same as the progress payments (PP) model, since in both the PP and TBPP payments are received at predefined moments in time in a regular manner (see e.g. Vanhoucke et al., 2003, Mika et al., 2005, Leyman and Vanhoucke, 2016).

In the example of Figure 2, we assume payments occur at time units 5 and 10. The resulting cash inflows at these times, which amount to the total value $\mathrm{TV}_{t}$ at the payment time minus any previous payments, are shown in the row $\mathrm{CF}_{\text {in,t,T }}$ (marked in bold), and their NPV in the corresponding row $\mathrm{NPV}_{\text {in }, t, \mathrm{~T}}$ below. The total NPV of the example for the TBPP is $75.88(=217.90-142.02)$.

\subsubsection{The progress-based payment pattern}

The progress-based payment pattern (PBPP) assumes payments occur once a threshold in terms of total value TV is exceeded. This threshold constitutes $\sum_{i=1}^{n} c_{i, \text { in }} / K$, and takes the cash inflows of activities into account. Since the payment times are, however, set based on whether or not at a certain point in time this threshold is exceeded, the timing depends on the project schedule. As a result, the timing of cash inflows cannot be determined in advance, but is set based on the TV progression in a specific schedule. The final payment is always received upon project completion. The size of payments is determined in the same manner as for the TBPP. 
In terms of the example, consider the $\mathrm{CF}_{\mathrm{in}, t, \mathrm{P}}$ and $\mathrm{NPV}_{\mathrm{in}, t, \mathrm{P}}$ rows in Table 4 . The threshold is set to $(160+72) / 2=116$, which is exceeded at time 3 . Hence, the first of two payments is received at that time and corresponds with the total value of 120 since the start of the project. The second payment is due at the finish time of activity 2 and constitutes 112. The total NPV of the example project is $75.78(=217.80-142.02)$.

\subsubsection{The expense-based payment pattern}

The expense-based payment pattern (EBPP) works similarly to the PBPP, but payment times are now set based on the progression in terms of TC. The threshold is determined by the average mode

cost or cash outflows for the activities and equals $\frac{\sum_{i=1}^{n} \sum_{m=1}^{\left|M_{i}\right|} c_{i m, \text { out }} /\left|M_{i}\right|}{K}$. As a result, the timing of payments again depends on the project schedule, but also on the TC progression in a specific schedule.

The $\mathrm{CF}_{\mathrm{in}, t, \mathrm{E}}$ and $\mathrm{NPV}_{\mathrm{in}, t, \mathrm{E}}$ rows in Table 4 display the cash flows and NPV of the payments received in the EBPP. The payment threshold equals 87.50 and results in payments at times 6 and 10. The project NPV equals $74.70(=216.72-142.02)$.

\section{An iterated local search with different solution representations}

Iterated local search (ILS) is a metaheuristic which operates on a single solution, and iteratively applies local search and perturbation operators. The local search aims to obtain a local optimum (intensification), whereas the perturbation step allows for the exploration of different regions in the solution space (diversification). For an overview of different ILS frameworks, along with practical applications, we refer to Lourenço et al. (2010).

The ILS framework used in this work is shown in Algorithm 1, and consists of four steps. A preprocessing method (PREPROCESSING () ) is applied at the start of the first step, to eliminate infeasible modes. We use the method of Akkan et al. (2005) to omit modes which, if selected, would always lead to an infeasible schedule with respect to the imposed deadline. To test this for each mode of an activity, we select the shortest possible mode for all other activities, and calculate the minimum project duration. If this total duration exceeds the project deadline, then the mode under consideration is infeasible and should not be evaluated in the ILS. To avoid excessive calculations, we always start with the shortest mode of an activity, such that if an infeasible mode is found, all modes with a longer duration can immediately be omitted as well, without the need for further calculations. After preprocessing, we randomly generate a number of solutions (\#Init) and retain the best one as both initial solution $x$ and currently best solution $x^{*}$. Additionally, the temperature $T$, used in the acceptance criterion, is set to its initial value $T_{0}$.

The next three steps constitute the actual ILS and consist of perturbation, evaluation \& local search, and acceptance criterion. The first two are discussed per representation below, whereas the 
last can be summarized as follows. If the neighboring solution $x^{\prime \prime}$ has a better objective function value $f\left(x^{\prime \prime}\right)$ than the current solution $x$, the latter is updated. Otherwise, the current solution $x$ may still be replaced by its neighbor $x^{\prime \prime}$ with probability $e^{\left(\frac{f\left(x^{\prime \prime}\right)-f(x)}{T}\right)}$. This acceptance criterion bears similarities to a simulated annealing algorithm and was first discussed by Martin et al. (1991). Additionally, the best solution $x^{*}$ is updated as well if it is worse than the neighbor $x^{\prime \prime}$. The current temperature $T$ is updated at the end of the acceptance criterion step. These three steps (perturbation, evaluation \& local search and acceptance criterion) are repeated until a stopping criterion has been met.

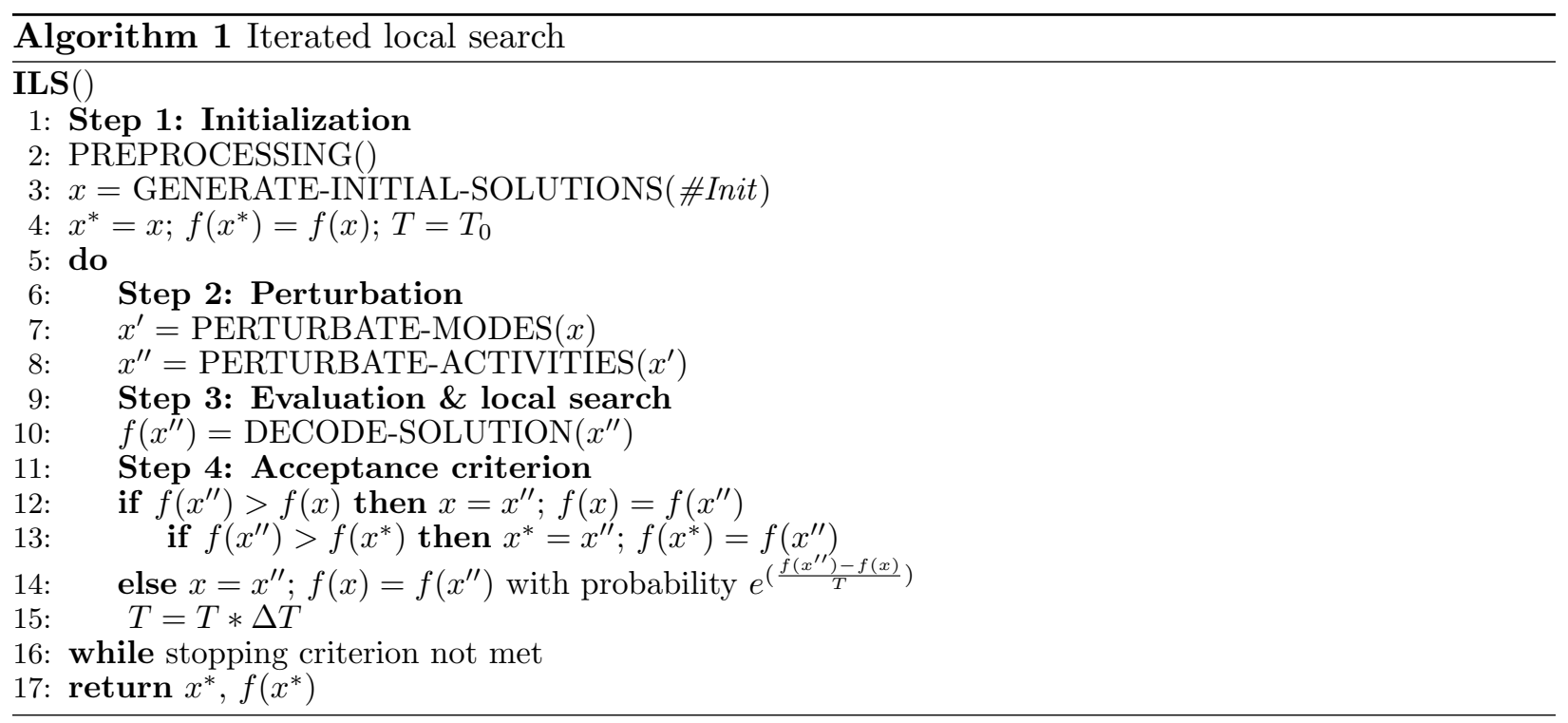

In order to represent a solution $x$ for the DTCTP-NPV, two types of lists are needed. The first is a mode list (ML), which holds the selected activity modes, and a second list, which contains information regarding the finish times of activities. We discuss the ML including its link to deadline feasibility, and several alternatives for the second list (i.e. the activity ordering). An overview of the decoding procedure is provided in Figure 3, with a distinction based on the representation used for the second list. Whereas the ML part of the procedure in Figure 3 is always applied, only one of the three other representation alternatives is used afterward.

The ILS discussed here differs from other metaheuristics in literature used for the DTCTP-NPV as follows:

- We change both the mode selection and activity ordering simultaneously, whereas most recent works use a double-loop metaheuristic in which the mode selection and activity ordering are altered in nested loops (Section 1). We believe this will allow the ILS to more easily escape local optima because the solution space can be traversed in a more flexible manner.

- We include an explicit decoding procedure, to translate each of the solution representations 
into an actual schedule. This is done to both highlight the impact of different representations and to show the importance of the corresponding decoding procedures.

Our results in Section 4 confirm the importance of both differences.

In the remainder of this section, we discuss each of the solution representations. We first go into detail about the characteristics of each representation and the decoding procedures, before explaining the perturbation operators for each type of representation. We distinguish between mode selection and activity ordering, with three alternatives for the latter. Finally, consider that the goal of this section is to provide a general framework, in which all representations can be properly used and compared, rather than proposing entirely new local search and perturbation operators.

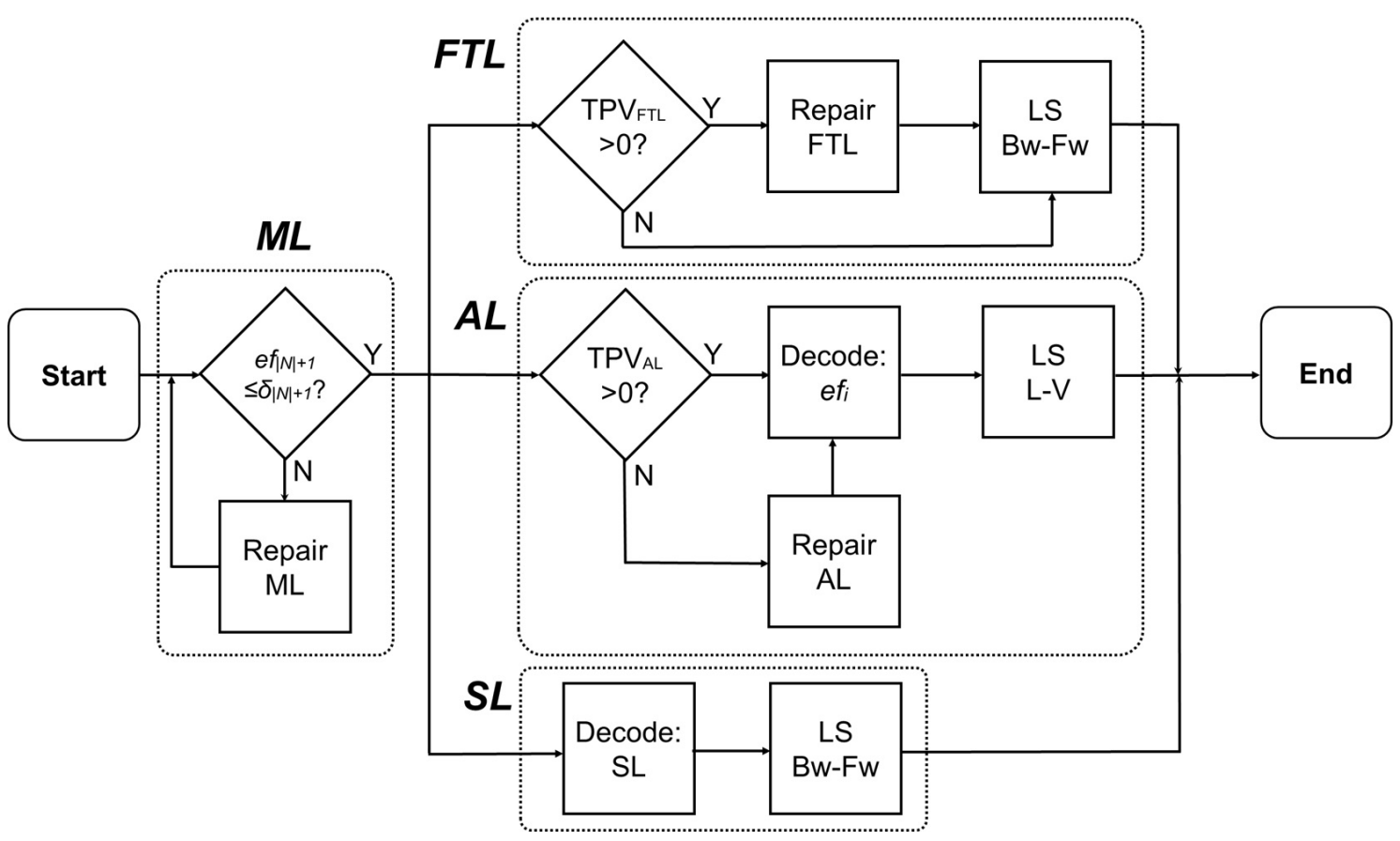

Figure 3: Flowchart DECODE-SOLUTION per representation.

\subsection{Mode selection}

The first solution representation handles the selection of modes for each activity. We always employ an ML to select a mode for each activity.

Decoding procedure: Since the ML determines the activity modes, it also determines the activity durations and costs. The selected modes may, however, be infeasible with respect to the project deadline. In such a case, we apply a deadline feasibility improvement method (Repair $M L$ in Figure 3). The mode of a random activity on the critical path is changed to the least expensive mode in terms of costs which allows for a reduction in project duration. The critical path and 
project duration are subsequently recalculated, and the improvement method is repeated until the project duration no longer exceeds the deadline.

Perturbation: In the perturbation step of the ML (PERTURBATE-MODES in Algorithm 1 ), we randomly assign a different mode to each activity with probability $R_{\mathrm{ML}}$. The probability is applied to each activity separately. In case the new ML is infeasible with respect to the project deadline, this infeasibility is later rectified in the decoding procedure, after perturbation has also been applied on the second list.

\subsection{Activity ordering}

We discuss three alternatives to determine the manner or order in which activities are scheduled, namely the activity list, the finish time list and the slack list. We discuss both the decoding procedures and perturbation operators for each of the three types of lists.

\subsubsection{Activity list}

The activity list (AL) representation holds the activity numbers in the order in which they should be scheduled. Precedence feasibility is of crucial importance for the AL, since it allows for easy scheduling, i.e. an activity can be scheduled without the need to take later activities in the AL into account.

Decoding procedure: We define the total precedence violation for an AL:

$$
\mathrm{TPV}_{\mathrm{AL}}=\sum_{i=1}^{n} \max \left(0 ; \max _{j \in P_{i}} b_{j}-b_{i}\right)
$$

with $b_{i}$ the position of activity $i$ in the AL and $P_{i}$ the set of predecessors of activity $i$. The $\mathrm{TPV}_{\mathrm{AL}}$ determines the total number of positions in the AL that activities are included before their predecessors. If this value is greater than zero, this means that some activities occur before at least one of their respective predecessors in the AL. In this case, we apply a straightforward repair method (Repair $A L$ in Figure 3). The method starts from the first activity and evaluates whether any of its predecessors are included in the AL after the current activity $i$. If this is the case, activity $i$ is moved immediately after the last predecessor in the list. The repair method then continues by evaluating the same position in the AL again, since it now contains a new activity number. Otherwise, the procedure continues with the next activity in the AL until the end of the list is reached. Once the repair method terminates, the AL is precedence feasible.

Since the AL only contains the priority of activities, but no information regarding the project schedule, we need a decoding procedure to determine activity finish times. As stated earlier in Section 1, the serial schedule generation scheme of Kelley (1963) is the most commonly used when resource restrictions apply as well. Since, however, no such constraints are included in the DTCTPNPV, we can initially schedule all activities at their earliest finish times, given the precedence 
restrictions and selected ML (Decode: $e f_{i}$ in Figure 3). As a result, the priority values provided by the $\mathrm{AL}$ are not taken into account in this initial schedule.

Once the early start schedule has been constructed, we apply the local search technique of Leyman and Vanhoucke (2016) ( $L S L$-V, short for Local Search of Leyman and Vanhoucke, in Figure 3). This local search was originally designed for the TBPP model in a resource-constrained context, and aims to delay (sets of) activities to increase project NPV. Since no renewable resource are included in the DTCTP-NPV, we only use the network-based moves of Leyman and Vanhoucke (2016). The procedure takes the precedence relations and activity finish times into account to determine which activities should be moved together, based on the occurrence of payment times. As a result, activities can be moved alone or together with other activities. The local search starts from the back of the AL, and is repeated until the front of the AL can be reached without any changes in activity finish times. One minor adjustment is made to the original procedure, in order to cope with the different cash flow cases (Section 2.1). If cash inflows are created entirely at activity start time (model 1), we adjust the local search such that delays are determined based on activity start times instead of finish times. This way, cash inflows can be scheduled as close as possible to payment times for this cash flow model as well.

A critical remark, however, has to be made regarding the local search and its application to the PBPP and EBPP models. Recall from Section 2.2 that in these models, unlike in the TBPP model, the payment times depend on the schedule, i.e. on the activity finish times. As a result, delaying activities may also delay payment times, resulting in a different NPV than expected. We illustrate this principle with the example of Figure 4. For the sake of simplicity, we employ only one mode for each activity. We use the PBPP model and cash case III from Table 2 (cash out at activity start time, cash in at activity finish time) and a discount rate $\alpha$ of $1 \%$. The project deadline is set to 10 and 3 payments occur. Since the TV, or the sum of all activity cash inflows, equals 240 , the payment threshold is set to $80(=240 / 3)$.

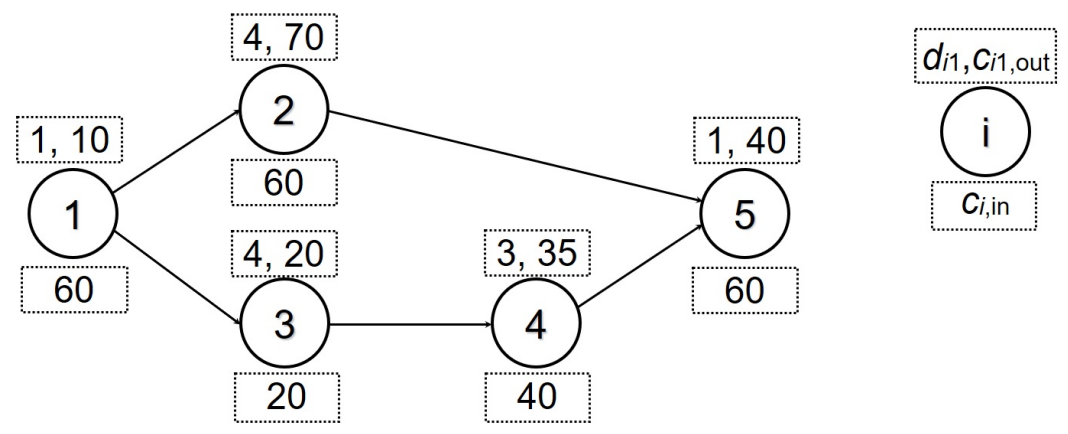

Figure 4: Example 2: Data project.

Table 5 displays the created value of each activity during the project. The values marked in bold are used to show the activity finish times, which correspond with the time instances at which 
the activity values are entirely realized. Based on the final row of the table, we can derive the times at which the payment threshold of 80 is exceeded, namely at times 2,7 and 10 . As a result, the project NPV equals 56.62 .

\begin{tabular}{r|ccccccccccc}
$t$ & 0 & 1 & 2 & 3 & 4 & 5 & 6 & 7 & 8 & 9 & 10 \\
\hline Act 1 & 0 & $\mathbf{6 0}$ & 60 & 60 & 60 & 60 & 60 & 60 & 60 & 60 & 60 \\
Act 2 & 0 & 0 & 15 & 30 & 45 & $\mathbf{6 0}$ & 60 & 60 & 60 & 60 & 60 \\
Act 3 & 0 & 0 & 5 & 10 & 15 & $\mathbf{2 0}$ & 20 & 20 & 20 & 20 & 20 \\
Act 4 & 0 & 0 & 0 & 0 & 0 & 0 & 13.33 & 26.67 & $\mathbf{4 0}$ & 40 & 40 \\
Act 5 & 0 & 0 & 0 & 0 & 0 & 0 & 0 & 0 & 0 & 0 & $\mathbf{6 0}$ \\
\hline Total & 0 & 60 & $\mathbf{8 0}$ & 100 & 120 & 140 & 153.33 & $\mathbf{1 6 6 . 6 7}$ & 180 & 180 & $\mathbf{2 4 0}$
\end{tabular}

Table 5: Example 2: Total created value per time instance before delay.

If we apply the local search discussed earlier, we would delay activity 2 from time 5 to time 7 , which would increase NPV from 56.62 to 57.28 , because the FT now equals the PT of 7 . This delay would, however, also alter the total created value for the project, as displayed in Table 6 . From the table, we can conclude that the first payment now occurs two time units later, namely at time 4 instead of at time 2. As a result, the project NPV only increases to 56.73 instead of 57.28. In this example, the delay of activity 2 was beneficial, in spite of the delay of the first payment time, but this can neither be generalized nor (easily) anticipated in advance. Consider the same example, but with a cost of 40 instead of 70 for activity 2. Tables 5 and 6 again apply, and only the project NPV changes. Initially, its value is 86.32 , it subsequently increases to 86.39 because of the delay of activity 2 , but decreases to 85.84 because of the change in the first payment time. Hence, the delay of activity 2 , somewhat counter-intuitively, turned out to be disadvantageous.

\begin{tabular}{r|ccccccccccc}
$t$ & 0 & 1 & 2 & 3 & 4 & 5 & 6 & 7 & 8 & 9 & 10 \\
\hline Act 1 & 0 & $\mathbf{6 0}$ & 60 & 60 & 60 & 60 & 60 & 60 & 60 & 60 & 60 \\
Act 2 & 0 & 0 & 0 & 0 & 15 & 30 & 45 & $\mathbf{6 0}$ & 60 & 60 & 60 \\
Act 3 & 0 & 0 & 5 & 10 & 15 & $\mathbf{2 0}$ & 20 & 20 & 20 & 20 & 20 \\
Act 4 & 0 & 0 & 0 & 0 & 0 & 0 & 13.33 & 26.67 & $\mathbf{4 0}$ & 40 & 40 \\
Act 5 & 0 & 0 & 0 & 0 & 0 & 0 & 0 & 0 & 0 & 0 & $\mathbf{6 0}$ \\
\hline Total & 0 & 60 & 65 & 70 & $\mathbf{9 0}$ & 110 & 138.33 & $\mathbf{1 6 6 . 6 7}$ & 180 & 180 & $\mathbf{2 4 0}$
\end{tabular}

Table 6: Example 2: Total created value per time instance after delay.

We can see that simply by altering the cost of a single activity, the delay of that activity may result in a deterioration instead of an improvement of the NPV. This effect cannot be anticipated by the local search of Leyman and Vanhoucke (2016), since it assumes fixed payment times. To mitigate this issue, we propose to recalculate the project NPV every time the local search finishes, such that at the end of the local search at least the correct NPV is reported, while limiting the required computation time. The shortcomings of the local search for the PBPP and EBPP are verified by our computational results in Section 4. 
Finally, the topological ordering (TO) can be considered an extension of the AL representation, since it adjusts the AL after all activities have been scheduled. In the TO, activities are ordered based on their finish times, with activities with a later finish time appearing later in the list. Ties in terms of activity finish times are broken randomly. Since the TO is an extension of the AL representation, we evaluate the impact of the TO on the results in Section 4, but do not discuss it here further nor treat it as an entirely different representation.

Perturbation: The perturbation operator for an AL (PERTURBATE-ACTIVITIES in Algorithm 1) is different from the operator for an ML, because we do not assign a new value per activity, but rather swap the position of two activities in the AL. With a probability $R_{\mathrm{AL}}$, the activity at each position can be swapped with a different activity at a different randomly selected position. Again, any infeasibilities as a result of the activity swaps, are solved in the subsequent decoding procedure (Repair $A L$ in Figure 3).

\subsubsection{Finish time list}

The finish time list (FTL) holds the finish times of all activities, ranging from each activity's respective earliest finish time to its respective latest finish time.

Decoding procedure: Since the FTL already contains the activity finish times, no scheduling procedure is needed to translate an FTL into a project schedule, since the schedule can immediately be determined. A downside of the FTL, however, is the possibility of precedence relation violations, i.e. an activity may start before its predecessors have been completed. The total predecessor violation $\left(\mathrm{TPV}_{\mathrm{FTL}}\right)$ can be calculated as follows:

$$
\operatorname{TPV}_{\mathrm{FTL}}=\sum_{i=1}^{n} \sum_{j=1}^{\left|P_{i}\right|} \max \left(0 ; f_{i}-d_{i m}-f_{j}\right)
$$

To the best of our knowledge, only Leyman (2016) proposes and tests two simple repair methods. We combine the best features of both methods into Algorithm 2 (Repair FTL in Figure 3), which is applied if the TPV FTL value of an FTL is greater than zero. The goal of Algorithm 2 is to ensure the FTL is precedence feasible, while minimizing the deviation from the infeasible FTL values. First, all activity finish times are randomly set at their earliest or latest possible times, $e f_{i}$ and $l f_{i}$ respectively (lines 2-6). Afterward, each activity's updated finish time $f_{i}$ is compared with the infeasible finish time $f_{i}^{\prime}$, and the activity is moved as close as possible to $f_{i}^{\prime}$ without violating precedence restrictions (lines 7-20). $S_{i}$ is the set of successors of activity $i$, whereas $P_{i}$ is the set of predecessors. Once all activities have been considered, this step is repeated until no more changes in activity finish times occur. The resulting schedule is always precedence feasible, but at the cost of possible deviations from the intended finish times based on the initial FTL.

Once a feasible schedule has been obtained, we apply a straightforward local search ( $L S B w-F w$ in Figure 3), which consists of two steps: 


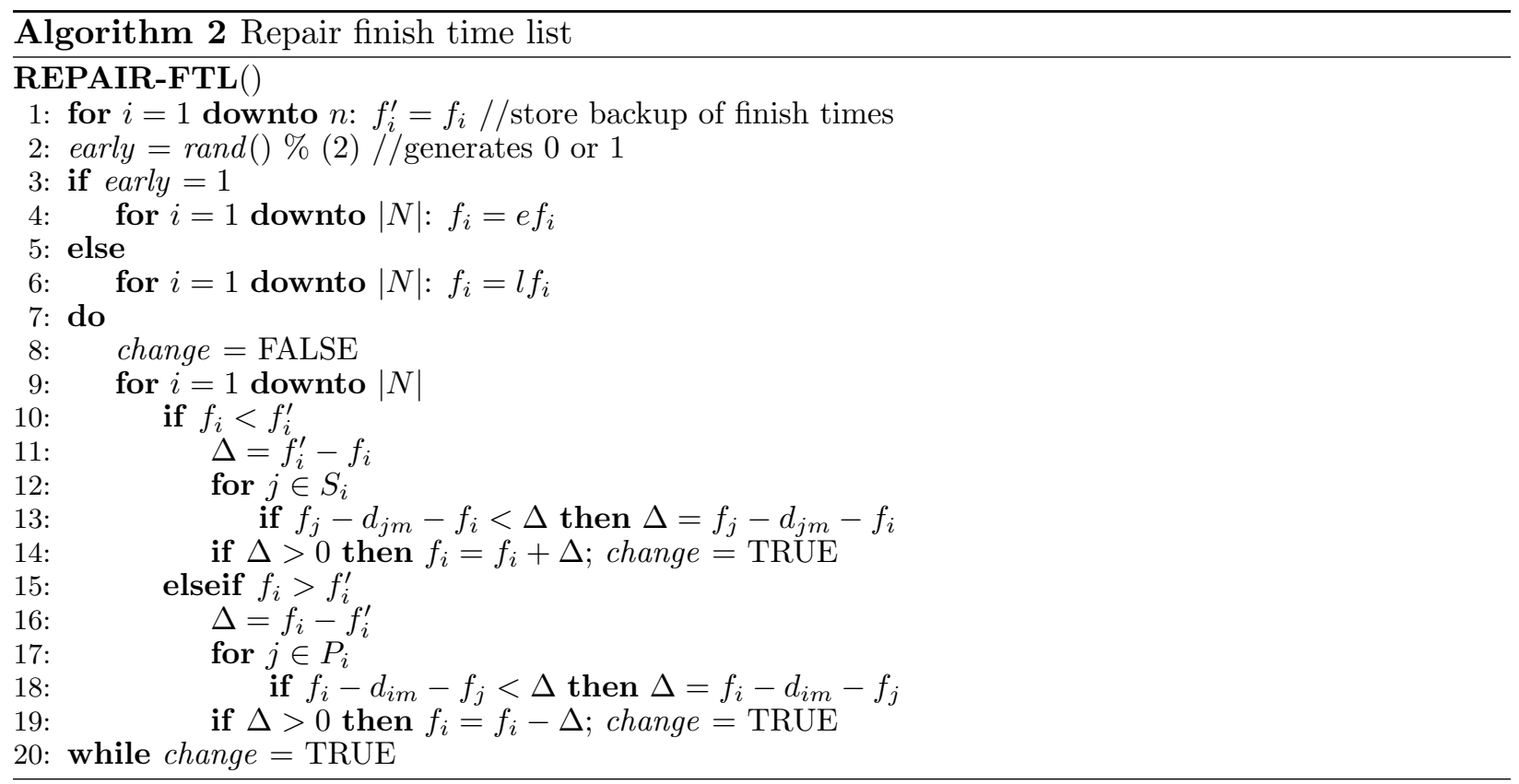

- Backward (Bw): We evaluate possible delays based on the precedence restrictions and occurrence of payment times, and determine the impact on the project NPV. Just like for the $\mathrm{L}-\mathrm{V}$ local search, payment times are assumed to be fixed, so the local search aims to move activities as close as possible to the earliest subsequent payment time. If the NPV can be increased, we apply the determined delay. This step corresponds with the delays of the local search in Section 3.2.1, with the major difference that in the earlier section both individual activities and sets of multiple activities could be delayed, whereas in this section we only delay single activities. The activities are considered for a delay in decreasing order of their finish times.

- Forward (Fw): The forward step is similar to the backward step, with the major difference that we now seek to advance activities, i.e. schedule activities earlier, starting from the activity with the earliest finish time.

The combination of both steps is repeated until the end dummy activity can be reached without any changes in activity finish times.

It can be observed that the FTL representation bears strong similarities to the shift vector (SV) proposed by Sampson and Weiss (1993). The SV sets activity finish times as a number of time units above the early finish times, e.g. activity $i$ may have an SV value of 2, resulting in a finish time of 2 time units later than its earliest possible finish time of 5. As a result, an FTL value can be translated into an SV value by subtracting the activity's $e f_{i}$. This implies that both representations only differ by a constant value (given the same ML), and hence contain the same information regarding the project schedule. In this paper, we use the FTL since the payment 
models of He et al. (2009b) are also implemented with the FTL representation.

Perturbation: For each activity, the perturbation step (PERTURBATE-ACTIVITIES in Algorithm 1) evaluates whether the activity slack is greater than zero. If this is indeed the case, a new value out of the interval $\left[e f_{i} ; l f_{i}\right]$ is assigned to each activity $i$, with a probability $R_{\text {FTL. Just }}$ like for the ML, any infeasibilities in the FTL resulting from the perturbation, are mitigated in the subsequent decoding procedure (Repair FTL in Figure 3).

\subsubsection{Slack list}

A slack list (SL) contains a value $r_{i}\left(r_{i} \in[0 ; 1[)\right.$ for each activity $i$, and determines the percentage of the available slack $s_{i}$ to be used.

Decoding procedure: The finish time for each activity is calculated as follows: $f_{i}=e f_{i}+$ $\left\lfloor\left(s_{i}+1\right) \cdot r_{i}\right\rfloor$. As an example, consider a project with three activities, of which only one remains to be scheduled. Based on the ML and the finish times of the other two activities, the remaining activity has an $e f_{i}$ equal to 5 and an $s_{i}$ equal to 4 . This results in five possible finish times, namely $5,6,7,8$ and 9 . Given an $r_{i}$ value of 0.61 , the finish time of the remaining activity is determined as $5+\lfloor(4+1) \cdot 0.61\rfloor=8$.

Algorithm 3 displays the decoding procedure for the SL, with $U$ the set of unscheduled activities and $F$ the set of finished or scheduled activities. The activity slack is updated dynamically for all activities in $U$ (lines 3-7) every time an activity is scheduled, taking into account the selected finish times for activities in $F$. As a result, no precedence violations occur, since the $e f_{i}$ and $s_{i}$ values are updated. In line 8 , the algorithm determines which activity should be scheduled next, namely the activity with the lowest $r_{i}$ value out of all unscheduled activities $U$. This way, both priority and schedule information are incorporated into the solution representation, i.e. the SL value for an activity determines the amount of activity slack used, as well as the priority or importance of each activity.

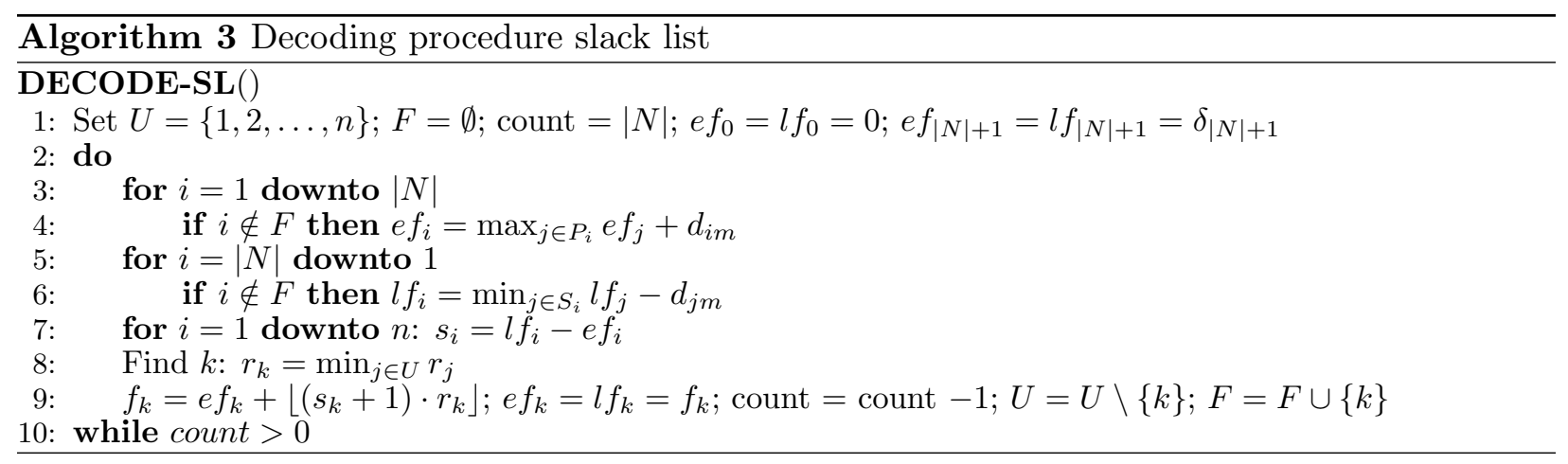

Once all activities have been scheduled, we apply $L S B w-F w$ in the exact same manner as for the FTL representation (Section 3.2.2). 
Perturbation: For the SL representation, the perturbation operator (PERTURBATE-ACTIVITIES in Algorithm 1) randomly assigns a different $r_{i}$ value $\left(r_{i} \in[0 ; 1[)\right.$ for each activity $i$, with a probability $R_{\mathrm{SL}}$. Unlike the perturbation operators for the other three representations (ML, AL and FTL), the obtained values for SL can never be infeasible, and hence no repair method as part of the decoding procedure is required.

\subsubsection{Summary}

Table 7 summarizes the differences between the three representations AL, FTL and SL. We can conclude that the SL is somewhat of a middle ground between the FTL and AL. The SL contains both schedule related information regarding activity finish times, i.e. the SL value for an activity determines the amount of activity slack used, as well as the priority or importance of each activity. The FTL and AL representation on the contrary contain only one of both. Unlike the FTL and AL, the SL furthermore never needs a repair method. Finally, the SL requires a less intricate decoding procedure than the local search used by the AL, but a less straightforward procedure than the one for the FTL.

\begin{tabular}{l|cc|cc} 
& Schedule & Priority & Feasible & Intricacy \\
\hline AL & & $\mathrm{X}$ & $?$ & High \\
FTL & $\mathrm{X}$ & & $?$ & Low \\
SL & $\mathrm{X}$ & $\mathrm{X}$ & $\mathrm{Y}$ & Medium
\end{tabular}

Table 7: Characteristics per representation.

\section{Results \& analysis}

In this section, we discuss the results of the ILS algorithm for the different solution representations. We first explain the test data employed and configure the proposed ILS for each solution representation, before comparing the results of the different representations in detail. As stopping criterion, we employ the 5000 schedules criterion by Lova et al. (2009). For the NPV calculations, we use a discount rate $\alpha$ of $1 \%$. All tests have been run in Visual Studio 2015 on a notebook computer with an i7-6820HQ $2.7 \mathrm{GHz}$ processor.

\subsection{Test data}

To generate project networks, we use the RanGen network generator of Demeulemeester et al. (2003). Network structures are generated in AoN format with four levels for the number of activities $(A c t)$ and three levels for the order strength $(O S)$. Based on three levels for the number of modes (Modes), we randomly generate the duration and cost (or cash outflow) values for the different activities ourselves $\left(d_{i m}, c_{i m, \text { out }} \in[1 ; 100]\right)$. For each of the combinations of these three parameters, 
we construct one (configuration) or five (comparison) instances. The project deadline is set as a value between the minimum and maximum project duration: $\delta_{|N|+1}=e f_{|N|+1}+D \cdot\left(l f_{|N|+1}-\right.$ $\left.e f_{|N|+1}\right) . \quad D$ is decimal value between 0 and 1 , and $e f_{|N|+1}$ and $l f_{|N|+1}$ are the earliest and latest finish times of the project, based on the shortest and longest activity modes respectively. The cash inflows are generated as follows: $c_{i, \text { in }}=\min _{m \in M_{i}} c_{i m, \text { out }}+P M \cdot\left(\max _{m \in M_{i}} c_{i m, \text { out }}-\min _{m \in M_{i}} c_{i m, \text { out }}\right)$, with $P M$ the profit margin $(P M \in[0 ; 1])$. Hence, the cash inflows are set as a value between the minimum and maximum cost of each activity. Note that we use a different dataset for the algorithm configuration (Section 4.2) than for the comparison between solution representations (Section 4.3). The former consists of one instance for each combination of the data parameters $(26,244$ instances in total), whereas the latter contains five such instances (131,220 instances in total). Both datasets are generated independently of one another.

An overview of the values for each of the data parameters is displayed in Table 8. The data presented here is available online at https://set.kuleuven.be/codes/dtctp-npv, along with the results of Section 4.3.

\begin{tabular}{ll}
\hline Parameter & Values this work \\
\hline Number of activities $($ Act $)$ & $25,50,75,100$ \\
Order strength $($ OS $)$ & $0.25,0.5,0.75$ \\
Number of modes $($ Modes $)$ & $2,4,6$ \\
Deadline increase $(D)$ & $0.25,0.5,0.75$ \\
Number of payments $(K)$ & $4,6,8$ \\
Profit margin $($ PM $)$ & $0.25,0.5,0.75$ \\
Cash flow cases $($ Cash $)$ & I-IX \\
Payment models $($ Pay $)$ & TBPP, PBPP, EBPP \\
\hline Total number of instances & $26,244 / 131,220$ \\
\hline
\end{tabular}

Table 8: Data parameter settings of test instances.

The generated test instances always allow for feasible solutions. Consider that the only problem restrictions are the precedence relations between activities and the project deadline (Section 2). Since the value of the latter is set between the minimum and maximum project duration, based on the minimum and maximum activity durations, we can always select activity modes in order to meet the deadline. Furthermore, the project duration is always calculated taking the precedence relations into account. As a result, the generated data always allows for at least one feasible solution, namely by selecting the shortest mode for each activity. In terms of cash flows, cash flow models and payment models, no further restrictions are imposed on the problem. Hence, the resulting test instances can always be solved.

\subsection{Algorithm configuration}

We first discuss the results of the configuration of the ILS parameters for each solution representation. We employed a design of experiments methodology (Montgomery, 2005) to test a subset of 
possible combinations based on the L'16 array. The best found values for the parameters are shown in Table 9, with $\mathrm{X}$ in $R_{\mathrm{X}}$ corresponding with $\mathrm{AL}$, FTL or SL respectively. We can conclude that there are only small differences between the three representations in terms of algorithm parameters.

\begin{tabular}{lccccc}
\hline & \#Init & $T_{0}$ & $\Delta T$ & $R_{\mathrm{ML}}$ & $R_{\mathrm{X}}$ \\
\hline AL & 7 & 700 & 0.99 & 0.027 & 0.010 \\
FTL & 12 & 350 & 0.87 & 0.012 & 0.006 \\
SL & 14 & 350 & 0.998 & 0.021 & 0.019 \\
\hline
\end{tabular}

Table 9: Algorithm parameters per solution representation.

Next, Table 10 shows the contribution of the different operators for the AL representation in terms of average project NPV $(A v N P V)$. We compare results for the proposed decoding scheme including the L-V local search, without any local search (NoLS), and with a local search which only delays single activities backward in time $(\mathrm{Bw})$ corresponding with the backward step of the local search of Section 3.2.2. We also test the impact of the alternative TO representation, and of removing the perturbation operator for the $\mathrm{ML}\left(\backslash P_{\mathrm{ML}}\right)$ and for the $\mathrm{AL}\left(\backslash P_{\mathrm{AL}}\right)$. The values shown between brackets are the p-values of a paired samples t-test between that alternative and the $\mathrm{L}-\mathrm{V}$ variant. We can conclude that there is an added value of employing a local search, but that there is no significant difference between the $\mathrm{L}-\mathrm{V}$ and $\mathrm{Bw}$ variant, showing that a more intricate local search has no added value for the DTCTP-NPV, unlike in a resource-constrained context. A similar conclusion applies for the TO representation; its results are not significantly different from those with the AL representation. Based on a $5 \%$ confidence interval, both perturbation operators improve the results, although the p-value for the AL perturbation is relatively high (0.0488).

\begin{tabular}{ccccccc}
\hline & L-V & NoLS & Bw & TO & $\backslash P_{\mathrm{ML}}$ & $\backslash P_{\mathrm{AL}}$ \\
\hline TBPP & 168.50 & 100.99 & 167.69 & 168.50 & -115.94 & 168.43 \\
PBPP & 236.47 & 196.50 & 236.87 & 236.50 & -78.50 & 236.44 \\
EBPP & 78.50 & 30.10 & 78.87 & 78.36 & -145.70 & 77.61 \\
\hline Overall & 161.16 & 109.20 & 161.14 & 161.12 & -113.38 & 160.83 \\
& & $(<0.0001)$ & $(0.9182)$ & $(0.8224)$ & $(<0.0001)$ & $(0.0488)$ \\
\hline
\end{tabular}

Table 10: Contribution of local search \& perturbation operators AL (AvNPV).

Tables 11 and 12 display the same results, but for the FTL and SL representations respectively. In general, the conclusions for both representations are the same, namely there is a significant contribution of the proposed backward-forward (Bw-Fw) local search, compared to the results without any local search, with only a backward local search, or with the L-V local search. The perturbation operators also have an added value, except for the FTL representation. The results indicate that the ILS' performance is not significantly different with the inclusion of an FTL perturbation, implying that the Bw-Fw local search handles neighborhood changes in the FTL sufficiently. 


\begin{tabular}{ccccccc}
\hline & Bw-Fw & NoLS & Bw & L-V & $\backslash P_{\text {ML }}$ & $\backslash P_{\text {FTL }}$ \\
\hline TBPP & 172.65 & 150.12 & 170.60 & 169.41 & -23.74 & 173.02 \\
PBPP & 218.55 & 188.88 & 201.52 & 199.40 & -3.79 & 219.99 \\
EBPP & 67.95 & 38.84 & 61.15 & 60.05 & -57.60 & 66.54 \\
\hline Overall & 153.05 & 125.95 & 144.42 & 142.95 & -28.38 & 153.18 \\
& & $(<0.0001)$ & $(<0.0001)$ & $(<0.0001)$ & $(<0.0001)$ & $(0.4599)$ \\
\hline
\end{tabular}

Table 11: Contribution of local search \& perturbation operators FTL ( $A v N P V)$.

\begin{tabular}{ccccccc}
\hline & Bw-Fw & NoLS & Bw & L-V & $\backslash P_{\mathrm{ML}}$ & $\backslash P_{\mathrm{SL}}$ \\
\hline TBPP & 177.26 & 159.97 & 170.11 & 169.38 & -21.19 & 165.76 \\
PBPP & 238.37 & 207.35 & 221.40 & 219.08 & 15.73 & 219.42 \\
EBPP & 84.01 & 68.80 & 73.64 & 70.61 & -47.67 & 60.10 \\
\hline Overall & 166.55 & 145.37 & 155.05 & 153.02 & -17.71 & 148.43 \\
& & $(<0.0001)$ & $(<0.0001)$ & $(<0.0001)$ & $(<0.0001)$ & $(<0.0001)$ \\
\hline
\end{tabular}

Table 12: Contribution of local search \& perturbation operators SL (AvNPV).

\subsection{Comparison of solution representations \& analysis}

Now that the ILS has been configured for the three representations, we can compare their performance. Table 13 presents the results for the three ILS variations (ILS-SL, ILS-AL \& ILS-FTL) and distinguishes based on the different data parameter settings. The results are shown in terms of $A v N P V$ and the percentage of instances for which the alternative performs as least at good as the best result obtained (\%Best). As a result, the sum of the \%Best of a row may sometimes be slightly larger than 1 , because more than one alternative obtained the best performance.

Additionally, we also compare with the double-loop simulated annealing (SA) algorithm of He et al. (2009b) (SA-FTL), to show the added value of changing both the mode selection (ML) and activity ordering (SL, AL or FTL). However, since the authors' data and code is not publicly available, we have chosen to implement the SA technique ourselves. To allow for a fair comparison, the following additions have been made to the original SA:

- The preprocessing method of Akkan et al. (2005) is included, to allow for the same mode elimination as in the ILS.

- In case a solution is infeasible with respect to the deadline, He et al. (2009b) always generate an entirely new solution. We adjust the SA such that the feasibility improvement method of Section 3.1 is used instead. Recall that the latter method changes the mode of a single randomly selected activity on the critical path to a shorter mode, and repeats this until the solution is feasible. This way, there is no need for generating an entirely new solution.

- In terms of precedence feasibility, we adjust the SA to include Algorithm 2 of Section 3.2.2, to ensure that violations of precedence restrictions are solved. He et al. (2009b), on the contrary, 


\begin{tabular}{lccccccccc}
\hline & \multicolumn{4}{c}{ ILS-SL } & \multicolumn{2}{c}{ ILS-AL } & \multicolumn{2}{c}{ ILS-FTL } & \multicolumn{2}{c}{ SA-FTL } \\
\hline \multirow{2}{*}{ Act } & & $A v N P V$ & \%Best & $A v N P V$ & \%Best & $A v N P V$ & \%Best & $A v N P V$ & $\% B e s t$ \\
& 25 & $\mathbf{1 0 0 . 0 9}$ & $\mathbf{5 7 . 2 5}$ & 94.69 & 21.50 & 92.89 & 20.61 & 67.11 & 0.98 \\
& 50 & $\mathbf{1 5 7 . 1 4}$ & $\mathbf{4 3 . 9 8}$ & 152.24 & 30.43 & 145.20 & 25.11 & 98.54 & 0.51 \\
& 75 & $\mathbf{1 9 5 . 7 0}$ & $\mathbf{3 6 . 0 6}$ & 189.05 & 34.31 & 180.19 & 29.43 & 98.75 & 0.19 \\
& 100 & $\mathbf{2 2 8 . 0 0}$ & 30.55 & 220.71 & $\mathbf{3 5 . 7 0}$ & 210.77 & 33.59 & 98.83 & 0.16 \\
\hline OS & 0.25 & $\mathbf{3 1 5 . 9 8}$ & $\mathbf{4 9 . 7 7}$ & 305.64 & 30.13 & 295.16 & 19.89 & 210.90 & 0.35 \\
& 0.50 & $\mathbf{1 5 9 . 4 9}$ & $\mathbf{4 4 . 6 8}$ & 154.47 & 32.60 & 141.96 & 22.31 & 79.71 & 0.52 \\
& 0.75 & $\mathbf{3 5 . 2 4}$ & 31.45 & 32.41 & 28.72 & 34.67 & $\mathbf{3 9 . 3 5}$ & -18.18 & 0.51 \\
\hline Modes & 2 & $\mathbf{9 5 . 7 8}$ & $\mathbf{4 2 . 8 6}$ & 77.87 & 18.75 & 92.71 & 38.35 & 13.19 & 0.11 \\
& 4 & $\mathbf{1 9 1 . 9 7}$ & $\mathbf{4 2 . 7 0}$ & 189.96 & 32.99 & 176.80 & 23.93 & 115.11 & 0.48 \\
& 6 & 222.95 & $\mathbf{4 0 . 3 3}$ & $\mathbf{2 2 4 . 6 9}$ & 39.72 & 202.28 & 19.28 & 144.13 & 0.79 \\
\hline D & 0.25 & $\mathbf{1 3 8 . 5 4}$ & $\mathbf{4 7 . 8 4}$ & 130.64 & 28.02 & 124.83 & 23.77 & 52.43 & 0.49 \\
& 0.50 & $\mathbf{1 8 6 . 5 0}$ & $\mathbf{4 0 . 8 7}$ & 181.27 & 30.77 & 173.85 & 27.93 & 112.75 & 0.52 \\
& 0.75 & $\mathbf{1 8 5 . 6 6}$ & $\mathbf{3 7 . 1 8}$ & 180.60 & 32.67 & 173.12 & 29.85 & 107.25 & 0.37 \\
\hline$K$ & 4 & $\mathbf{1 1 1 . 3 8}$ & $\mathbf{3 6 . 5 2}$ & 106.14 & 31.41 & 98.98 & 31.95 & 21.30 & 0.22 \\
& 6 & $\mathbf{1 7 9 . 9 4}$ & $\mathbf{4 2 . 8 6}$ & 173.26 & 30.56 & 166.18 & 26.17 & 101.95 & 0.5 \\
& 8 & $\mathbf{2 1 9 . 3 8}$ & $\mathbf{4 6 . 5 1}$ & 213.11 & 29.48 & 206.63 & 23.43 & 149.18 & 0.66 \\
\hline PM & 0.25 & $\mathbf{1 9 . 4 0}$ & $\mathbf{4 2 . 7 0}$ & 2.86 & 18.16 & 19.21 & 38.94 & -50.45 & 0.26 \\
& 0.50 & $\mathbf{1 6 3 . 0 4}$ & $\mathbf{4 0 . 2 8}$ & 158.59 & 32.82 & 151.53 & 26.59 & 85.30 & 0.44 \\
& 0.75 & 328.26 & $\mathbf{4 2 . 9 1}$ & $\mathbf{3 3 1 . 0 6}$ & 40.46 & 301.05 & 16.02 & 237.58 & 0.69 \\
\hline Pay & TBPP & $\mathbf{1 8 1 . 8 5}$ & $\mathbf{4 8 . 1 8}$ & 173.04 & 22.26 & 178.34 & 29.71 & 110.44 & 0.10 \\
& PBPP & $\mathbf{2 4 1 . 8 0}$ & $\mathbf{4 6 . 1 8}$ & 239.63 & 33.79 & 221.81 & 19.84 & 160.35 & 0.21 \\
& EBPP & $\mathbf{8 7 . 0 4}$ & 31.53 & 79.85 & $\mathbf{3 5 . 4 0}$ & 71.63 & 32.00 & 1.64 & 1.07 \\
\hline Overall & & $\mathbf{1 7 0 . 2 3}$ & $\mathbf{4 1 . 9 6}$ & 164.17 & 30.48 & 157.26 & 27.18 & 90.81 & 0.46 \\
& & & & $(<0.0001)$ & & $(<0.0001)$ & & $(<0.0001)$ & \\
\hline
\end{tabular}

Table 13: Comparison of different approaches.

use a method which changes the finish times of activities, but offers no guarantee of finding a feasible solution, unlike our method. The authors rather again generate an entirely new solution in case of infeasibilities. Additionally, the method of He et al. (2009b) may lead to violations of the project deadline, whereas Algorithm 2 always respects the deadline.

- The same stopping criterion of 5000 schedules is used, instead of a criterion based on the temperature of the SA's outer loop. As a result, we have extended the SA such that its outer temperature loop is repeated, once the loop's stopping criterion is reached.

- We have configured the parameters of the SA in the same manner as for the ILS in Section 4.2. The parameter names as used by He et al. (2009b), their description, and the best found values are displayed in Table 14.

Based on the results in Table 13, we conclude the following:

- In general, the SL alternative significantly outperforms the other two alternatives. This shows that it is worthwhile to incorporate both schedule and priority information into the representation for the problem studied, thus allowing the ILS to take both types of information into account during its search. Only in the case of 6 modes and a high profit margin (0.75) 


\begin{tabular}{lll}
\hline Parameter & Description & Value \\
\hline Init neighbors & Number of neighbors of initial solution & 50 \\
Prob & Used to calculate the initial temperature & 0.87 \\
Cooling rate & Determines temperature decrease & 0.99 \\
Markov chain length & Number of neighbors generated for fixed temperature & 2 \\
Stop criterion & End temperature for any loop of the SA & 0.09 \\
\hline
\end{tabular}

Table 14: Algorithm parameters SA.

does the AL perform slightly better $(A v N P V)$. The results in terms of \%Best lead to the same conclusions in general, with some minor variations.

- The FTL representation performs worst, which is surprising, given that it is commonly used in DTCTP-NPV literature, albeit with an AoA network representation instead of the AoN used here. Furthermore, the AL has a better performance because it assigns a priority to all activities and only considers delays in the local search, even though it is typically employed for resource-constrained problems. The FTL instead holds no priority, making it more difficult for the local search to determine which activities should be advanced and which ones delayed, and in what order. As a result, the local search for the AL has more information than it does for the FTL, which leads to a better performance for the former.

- The effects of the data parameters on the project NPV are in general as expected, e.g. more payments $(K)$ and a higher profit margin $(P M)$ both lead to a higher NPV, whereas a more serial network structure (higher $O S$ ) decreases NPV. The only notable difference is the project deadline $(D)$, of which an increase first improves NPV, but no more improvement occurs as $D$ increases further. Given that the final payment is received either upon project completion (PBPP and EBPP) or at the deadline (TBPP), it makes sense that the contractor does not benefit from a large project deadline.

- The effect of the three payment models is in line with the overall performance of the three representations, namely the SL leads to the best performance, regardless of the payment model.

- The SA (SA-FTL) performs worst out of all options. We believe this is because the SA changes the FTL and ML in two nested loops, i.e. for a given ML the FTL neighborhood is searched, after which a change in the ML occurs and the process for the FTL is repeated. The ILS, on the contrary, makes changes to both types of lists simultaneously, allowing for a more flexible search of the solution space.

In Table 15 more details are provided for each of ILS' representations in terms of cash flow models. The values without brackets are the $A v N P V$, whereas the values between brackets are the \%Best. Table 15 paints a different picture than the results in Table 13, since it appears that 
the cash flow models have a strong impact on the representation to be preferred. Recall from the previous table, that this is in strong contrast with the effect of the payment models.

- In case cash inflows are received at the start of each activity (cash in model 1), the results for AL are best. This is because the AL, unlike the SL and FTL, starts from an early start schedule, which is beneficial if the cash inflows are received at the same time as or earlier than the cash outflows. A similar logic applies for the entire bottom row of the table, though the differences between the results for SL and AL are small in all cases.

- In case cash outflows are to be paid ahead of the receipt of cash inflows, AL performs worst, and either FTL (cash out model 1) or SL (cash out models $2 \& 3$ ) leads to the best results. In particular, the results for FTL are best in case the contractor has to prefinance all expenses, i.e. if cash outflows occur at the start of the activity and cash inflows are only received later. In such cases, starting from an early start schedule would be detrimental, rather than beneficial.

- SL appears to be most stable, since it consistently performs at least second best, which makes it the best solution representation on average, in line with the results in Table 13. However, the results in both tables show that the decision on which solution representation to use should not be made lightly, especially in terms of cash flow models.

\begin{tabular}{cc|ccc|ccc|ccc} 
& & \multicolumn{4}{|c}{ Model 1 } & \multicolumn{4}{c}{ Cash in } \\
& & Sodel 2 & \multicolumn{3}{c}{ Model 3 } \\
\hline & & SL & AL & FTL & SL & AL & FTL & SL & AL & FTL \\
\hline & Model 1 & 277.59 & $\mathbf{2 8 6 . 6 8}$ & 248.26 & 16.52 & -13.31 & $\mathbf{2 3 . 5 4}$ & -47.03 & -85.63 & $\mathbf{- 3 2 . 1 3}$ \\
& & $(31.62)$ & $(\mathbf{4 5 . 0 6 )}$ & $(23.42)$ & $(42.73)$ & $(9.08)$ & $(\mathbf{4 8 . 2 0})$ & $(34.09)$ & $(8.76)$ & $(\mathbf{5 7 . 1 5})$ \\
Cash out & Model 2 & 343.15 & $\mathbf{3 5 2 . 6 6}$ & 304.23 & $\mathbf{1 1 7 . 2 1}$ & 106.70 & 110.14 & $\mathbf{3 3 . 9 4}$ & 17.87 & 32.61 \\
& & $(35.43)$ & $\mathbf{( 4 9 . 0 5 )}$ & $(15.67)$ & $\mathbf{( 5 6 . 9 9 )}$ & $(16.62)$ & $(26.49)$ & $(\mathbf{4 5 . 6 4 )}$ & $(17.65)$ & $(36.75)$ \\
& \multirow{4}{*}{ Model 3 } & 443.38 & $\mathbf{4 6 0 . 7 3}$ & 407.63 & 215.58 & $\mathbf{2 1 7 . 2 9}$ & 201.79 & 131.75 & $\mathbf{1 3 4 . 5 4}$ & 119.30 \\
& & $(33.46)$ & $\mathbf{( 5 6 . 5 8 )}$ & $(10.16)$ & $\mathbf{( 5 4 . 5 7 )}$ & $(33.55)$ & $(12.03)$ & $(\mathbf{4 4 . 8 5})$ & $(39.56)$ & $(15.67)$
\end{tabular}

Table 15: Comparison different representations in terms of cash flow models (AvNPV, \%Best).

Finally, Table 16 shows the average computation time $(A v C T)$ in seconds, with details for Act and for Pay. SL has the highest computation time on average and FTL the lowest. As could be expected, the number of activities to schedule has a large impact on the computation time, whereas the payment model used also leads to differences. PBPP and EBPP have longer computation times, since both require the algorithm to recalculate the NPV after each application of the local search.

\section{Conclusions \& future research}

In this paper, we have discussed the discrete time/cost trade-off problem with net present value optimization (DTCTP-NPV). We have included three payment models from literature, which de- 


\begin{tabular}{lcccc}
\hline & & SL & AL & FTL \\
\hline Act & 25 & 0.48 & 0.53 & 0.54 \\
& 50 & 1.04 & 1.05 & 0.89 \\
& 75 & 1.80 & 1.65 & 1.20 \\
& 100 & 2.74 & 2.34 & 1.53 \\
\hline Pay & TBPP & 1.08 & 0.86 & 0.69 \\
& PBPP & 1.69 & 1.63 & 1.18 \\
& EBPP & 1.78 & 1.68 & 1.25 \\
\hline Overall & & 1.52 & 1.39 & 1.04 \\
\hline
\end{tabular}

Table 16: Computation times different representations $(A v C T)$.

termine the timing and/or size of payments from the client to the contractor. Taking the contractor point of view, we have extended these models by also incorporating different cash flow models. The cash flow models regulate how both the total value created for the client and the total cost incurred by the contractor progress during the project.

To solve the problem, we have employed an iterated local search (ILS) metaheuristic, with different solution representations and corresponding decoding procedures, including several local search heuristics. We have incorporated the representations into the overarching ILS framework, to allow for a comparison in performance. Based on an extensive computational experiment, we have configured the ILS for all three representations. We have shown that there are indeed significant differences in performance, highlighting the importance of considering which solution representation to use, and testing the impact of several alternatives. Specifically, our results indicate that the incorporation of both schedule and priority information into the solution representation, as part of the slack list alternative, was beneficial. Additionally, the ILS approach has been favorably compared with an approach from literature.

These results could in a sense be expected, but it is our belief that the choice of solution representation, as a metaheuristic component, currently does not (always) receive the attention it deserves. Rather, we urge researchers to also test and analyze several alternatives for the solution representation, as is (more) commonly carried out for intensification and diversification operators. In doing so, our understanding can be improved and valuable insights can be gained, which are after all crucial goals of academic research.

In the future, we want to include learning as an integral part of the metaheuristic framework, to allow for the algorithm to learn both from the data generated during algorithm execution, as well as from the test instance data. From a problem viewpoint, capital constraints, which ensure the cash balance of the contractor never becomes negative, can be introduced to avoid capital shortages. Finally, investigating the actual negotiation process with the client and/or subcontractor, could be worthwhile to model the interaction process and determine how project specifications regarding timing and size of payments impact performance. In such a case, it would be advantageous to optimize the project schedule and payment decisions for all parties involved. 


\section{Acknowledgments}

We acknowledge the support provided by the "Bijzonder Onderzoeksfonds" (BOF) and by the National Bank of Belgium (NBB) for the project with contract number BOF12GOA021, and by the Belgian Science Policy Office (BELSPO) in the Interuniversity Attraction Pole COMEX.

\section{References}

Akkan, C., Drexl, A., and Kimms, A. (2005). Network decomposition-based benchmark results for the discrete time-cost tradeoff problem. European Journal of Operational Research, 165:339-358.

Burke, E. and Kendall, G. (2014). Search methodologies - Introductory tutorials in optimization and decision support techniques - Second edition. Springer.

Demeulemeester, E., Vanhoucke, M., and Herroelen, W. (2003). RanGen: A random network generator for activityon-the-node networks. Journal of Scheduling, 6:17-38.

Erengüç, S., Tufekci, S., and Zappe, C. (1993). Solving time/cost trade-off problems with discounted cash flows using generalized Benders decomposition. Naval Research Logistics, 40:25-50.

Gendreau, M. and Potvin, J.-Y. (2010). Handbook of metaheuristics - Second edition. Springer.

Hartmann, S. and Briskorn, D. (2010). A survey of variants and extensions of the resource-constrained project scheduling problem. European Journal of Operational Research, 207:1-14.

Hazir, O., Haouari, M., and Erel, E. (2010). Discrete/time cost trade-off problem: A decomposition-based solution algorithm for the budget version. Computers \& Operations Research, 37:649-655.

He, Z., He, H., Liu, R., and Wang, N. (2017). Variable neighbourhood search and tabu search for a discrete time/cost trade-off problem to minimize the maximal cash flow gap. Computers \& Operations Research, 78:564-577.

He, Z., Liu, R., and Jia, T. (2012). Metaheuristics for multi-mode capital-constrained project payment scheduling. European Journal of Operational Research, 223:605-613.

He, Z., Liu, R., and Xu, Y. (2009a). Client perspective based multimode project payment scheduling problem and its heuristic algorithm. Systems Engineering - Theory \& Practice, 29(2):70-77.

He, Z., Wang, N., Jia, T., and Xu, Y. (2009b). Simulated annealing and tabu search for multi-mode project payment scheduling. European Journal of Operational Research, 198:688-696.

He, Z., Wang, N., and Li, P. (2014). Simulated annealing for financing cost distribution based project payment scheduling from a joint perspective. Annals of Operations Research, 213:203-220.

He, Z. and Xu, Y. (2008). Multi-mode project payment scheduling problem with bonus-penalty structure. European Journal of Operational Research, 189:1191-1207.

Kelley, J. J. (1963). The critical-path method: Resources planning and scheduling. In Muth, J. and Thompson, J., editors, Industrial Scheduling, pages 347-365. Prentice-Hall.

Kolisch, R. and Hartmann, S. (1999). Heuristic algorithms for solving the resource- constrained project scheduling problem: Classification and computational analysis. European Journal of Operational Research, 112:3-41. 
Leyman, P. (2016). Heuristic algorithms for payment models in project scheduling. PhD Dissertation (Ghent University). University Press.

Leyman, P. and Vanhoucke, M. (2015). A new scheduling technique for the resource-constrained project scheduling problem with discounted cash flows. International Journal of Production Research, 53(9):2771-2786.

Leyman, P. and Vanhoucke, M. (2016). Payment models and net present value optimization for resource-constrained project scheduling. Computers 6 Industrial Engineering, 91:139-153.

Leyman, P. and Vanhoucke, M. (2017). Capital- and resource-constrained project scheduling with net present value optimization. European Journal of Operational Research, 256(3):757-776.

Li, H., Xiong, L., Liu, Y., and Li, H. (2018). An effective genetic algorithm for the resource levelling problem with generalised precedence relations. International Journal of Production Research, 56:2054-2075.

Lourenço, H., Martin, O., and Stützle, T. (2010). Iterated local search: Framework and applications. In Gendreau, M. and Potvin, J.-Y., editors, Handbook of metaheuristics - Second edition, pages 363-397. Springer.

Lova, A., Tormos, P., Cervantes, M., and Barber, F. (2009). An efficient hybrid genetic algorithm for scheduling projects with resource constraints and multiple execution modes. International Journal of Production Economics, $117: 302-316$.

Martin, O., Otto, S., and Felten, E. (1991). Large-step Markov chains for the traveling salesman problem. Complex Systems, 5(3):299-326.

Mika, M., Waligóra, G., and Weglarz, J. (2005). Simulated annealing and tabu search for multi-mode resourceconstrained project scheduling with positive discounted cash flows and different payment models. European Journal of Operational Research, 164:639-668.

Montgomery, D. (2005). Design and Analysis of Experiments. John Wiley and Sons Inc.

Ning, M., He, Z., Jia, T., and Wang, N. (2017). Metaheuristics for multi-mode cash flow balanced project scheduling with stochastic duration of activities. Automation in Construction, 81:224-233.

Sampson, S. and Weiss, E. (1993). Local search techniques for the generalized resource constrained project scheduling problem. Naval Research Logistics, 40:665-675.

Sörensen, K. (2015). Metaheuristics-the metaphor exposed. International Transactions in Operational Research, 22:3-18.

Sörensen, K. and Glover, F. (2013). Metaheuristics. In Gass, S. and Fu, M., editors, Encyclopedia of Operations Research and Management Science, pages 960-970. Springer, London.

Valls, V., Quintanilla, S., and Ballestín, F. (2003). Resource-constrained project scheduling: A critical activity reordering heuristic. European Journal of Operational Research, 149:282-301.

Van Peteghem, V. and Vanhoucke, M. (2011). Using resource scarceness characteristics to solve the multi-mode resource-constrained project scheduling problem. Journal of Heuristics, 17:705-728.

Vanhoucke, M. and Debels, D. (2007). The discrete time/cost trade-off problem: extensions and heuristic procedures. Journal of Scheduling, 10:311-326.

Vanhoucke, M., Demeulemeester, E., and Herroelen, W. (2003). Progress payments in project scheduling problems. European Journal of Operational Research, 148:604-620. 